\title{
Theories of Electromagnetic Productions of Pions
}

T.-S. H. Lee ${ }^{*}$

Physics Division, Argonne National Laboratory, Argonne, Illinois 60439, USA

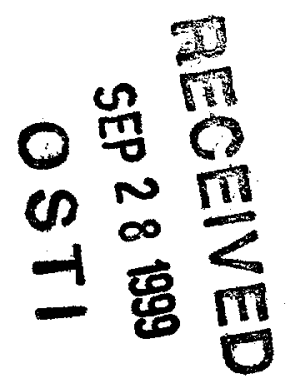

\begin{abstract}
The current theoretical approaches for investigating the $\gamma N \rightarrow \pi N$ and $N\left(e, e^{\prime} \pi\right)$ reactions are reviewed. It is shown that the dynamical approach can be used to test the predictions from hadron structure calculations. Some $N^{*}$ form factors calculated from using various chiral constituent quark models are presented.
\end{abstract}

\section{Introduction}

The opportunities for using the electromagnetic probes to explore the nonperturbative QCD aspects of hadron and nuclear dynamics will be unprecedented in the next few years, mainly owing to the developments of several new electron beam facilities. All of the existing theoretical studies of $\pi N$ and $\gamma N$ reactions can be classified into two different formulations. The first one is the dispersionrelations approach. In this approach, the dynamics is defined by imposing the crossing symmetry, analyticity, high-energy behavior, and appropriate subtraction terms on the reaction amplitudes. This approach was developed by Chew, Goldberger, Low and Nambu[1] in 1950's and has been revived recently by the Mainz group[2]. The second formulation is based on effective lagrangians. Within this framework, there are three different approaches. The first one is the K-matrix approach[3] which uses only the $\pi N$ scattering phase shifts to account for the $\pi N$ final state interactions. The second one is the Chiral Perturbation Theory[4] which is applicable mainly at energies near thresholds and for the low momentum-transfer processes. The third approach is the dynamical approach[5] which is aimed at testing the QCD-based hadron structure models. In this talk I will focus on the development of the dynamical approach and present our recent results[6].

- This work is supported by the U.S. Department of Energy, Nuclear Physics Division, under Contract No. W-31-109-ENG-38 


\section{DISCLAIMER}

This report was prepared as an account of work sponsored by an agency of the United States Government. Neither the United States Government nor any agency thereof, nor any of their employees, make any warranty, express or implied, or assumes any legal liability or responsibility for the accuracy, completeness, or usefulness of any information, apparatus, product, or process disclosed, or represents that its use would not infringe privately owned rights. Reference herein to any specific commercial product, process, or service by trade name, trademark, manufacturer, or otherwise does not necessarily constitute or imply its endorsement, recommendation, or favoring by the United States Government or any agency thereof. The views and opinions of authors expressed herein do not necessarily state or reflect those of the United States Government or any agency thereof. 


\section{DISCLAIMER}

Portions of this document may be illegible in electronic image products. Images are produced from the best available original document. 


\section{Dynamical Approach}

The starting point of a dynamical approach is a hadron structure calculation which predicts the masses of the excited states of the nucleon. In practice, this calculation can be done accurately only in the absence of the couplings with the decay channels. These masses are therefore called the bare masses which do not correspond to the resonance positions determined in the empirical amplitude analyses.

The next step is to introduce the couplings of these bare states with the meson and photon fields. In Ref.[5], we focus on the $N \rightarrow \Delta$ transition. By including the interactions with $\pi, \rho, \omega$ and $\gamma$ fields and applying a unitary transformation method, we obtain an effective Hamiltonain for describing $\pi N$ and $\gamma N$ reactions up to the $\Delta$ excitation energy region. It takes the following form:

$$
H_{e f f}=H_{0}+\sum_{\alpha=\gamma N, \pi N} \Gamma_{\Delta \leftrightarrow \alpha}+\sum_{\alpha, \beta=\gamma N, \pi N} v_{\alpha, \beta}
$$

where the two-body interactions $v_{\alpha \beta}$ are defined by the meson-exchange mechanisms. It is straightforward to derive from Eq.(1) the scattering amplitudes for $\pi N \rightarrow \pi N$ and $\gamma N \rightarrow \pi N$ reactions. The resulting scattering amplitudes can be cast into the following form

$$
T_{\alpha, \beta}(E)=t_{\alpha, \beta}(E)+\bar{\Gamma}_{\alpha \rightarrow \Delta}(E) \frac{1}{E-m_{\Delta}-\Sigma_{\Delta}(E)} \bar{\Gamma}_{\Delta \rightarrow \beta}(E) .
$$

The first term is the nonresonant amplitude which is generated from a relativistic Lippman-Schwinger equation with the two-body meson-exchange interactions $v_{\alpha, \beta}$. The second term of Eq. (2) is the resonant amplitude determined by the dressed $\Delta$ mass and the dressed vertex functions. They are defined by

$$
\begin{aligned}
& \bar{\Gamma}_{\pi N \rightarrow \Delta}(E)=\Gamma_{\pi N \rightarrow \Delta}+t_{\pi N, \pi N}(E) G_{\pi N}(E) \Gamma_{\pi N \rightarrow \Delta}, \\
& \bar{\Gamma}_{\gamma N \rightarrow \Delta}(E)=\Gamma_{\gamma N \rightarrow \Delta}+v_{\gamma N, \pi N}(E) G_{\pi N}(E) \bar{\Gamma}_{\pi N \rightarrow \Delta}(E),
\end{aligned}
$$

where $G_{\pi N}(E)$ is the free $\pi N$ propagator and

$$
\Sigma_{\Delta}(E)=\Gamma_{\pi N \rightarrow \Delta} G_{\pi N}(E) \bar{\Gamma}_{\Delta \rightarrow \pi N}(E) .
$$

In Ref.[5], we did not rely on a hadron structure calculation. Therefore the bare mass of the $\Delta$ and the form factors of the effective Hamiltonian are treated phenomenologically. Our first task was to determine the parameters of the hadronic part of the effective Hamiltonian, Eq.(1), by fitting the $\pi N$ phase shifts. Our results were presented and discussed in Ref.[5]. For this talk, it is only necessary to emphasize that the resulting bare mass of the $\Delta$ is $\sim 1300$ $\mathrm{MeV}$ which is considerably higher than the resonance position $1236 \mathrm{MeV}$ of the $\pi N$ cross section data.

We now turn to discussing our results of pion photoproduction. Here we also need to include the $\omega$-exchange which does not play a role in $\pi N$ scattering. Since the coupling constant $g_{\omega N N}$ is not well determined in the literature, it 
is treated as a free parameter in our study. Thus, our investigation of pion photoproduction has three adjustable parameters: $G_{M}(0)$ and $G_{E}(0)$ of the bare $\Delta \leftrightarrow \gamma N$ vertex, and the coupling constant $g_{\omega N N}$ of $\omega$ exchange. With $G_{M}(0)=1.85, G_{E}(0)=+0.025$ and $g_{\omega N} N=10.5$, we find that all of the available $\gamma N \rightarrow \pi N$ data can be described very well, as illustrated in Fig.1 for the LEGS data[9]. We also find that the data can be fitted equally well with $G_{M}(0)=1.95, G_{E}(0)=-0.025$ and $g_{\omega N N}=7.0$.

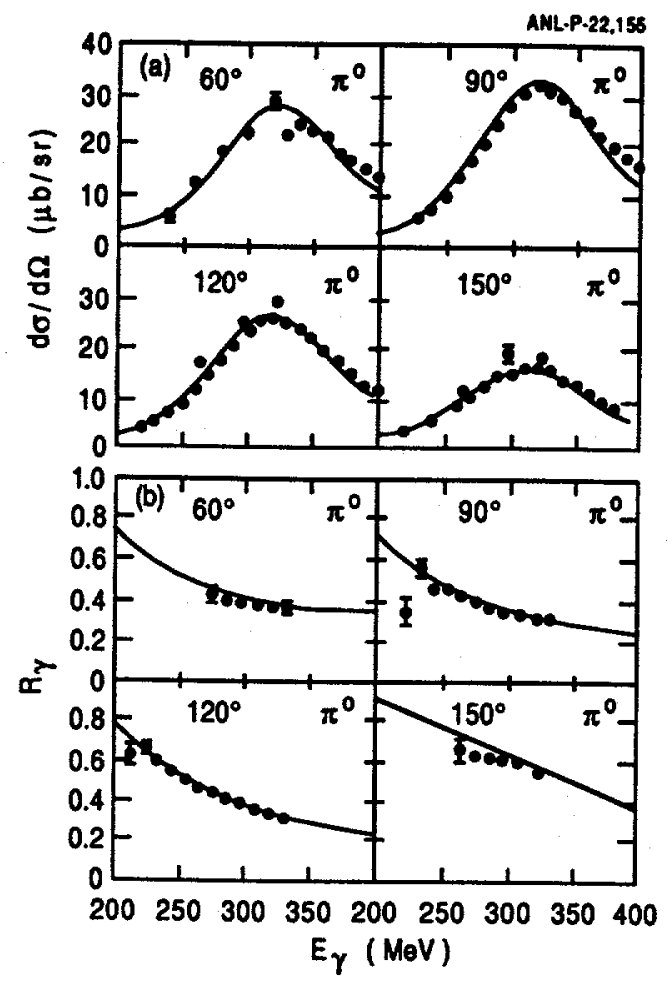

Fig.1 The calculated differential cross sections $d \sigma / d \Omega$ and $R_{\gamma}=d \sigma_{\|} / d \sigma_{\perp}$ of $\gamma p \rightarrow \pi^{0} p$ are compared with the LEGS data.

We now focus on the theoretical interpretations of the $\Delta \leftrightarrow \gamma N$ vertex. Eq.(2) indicates that it could be misleading to compare the branching ratios predicted by the constituent quark models $[7,8]$ with the values extracted from the amplitude analyses. This can be seen by comparing in Table 2.1 the predicted helicity amplitudes with the values listed by the Particle Data Group(PDG)[10]. We notice that our bare values are close to the constituent quark model predictions[7, 8], and the dressed values are close to the values of PDG[10]. This suggests that the large differences between the constituent quark model predictions and the PDG values are due to the nonresonant mesonexchange production mechanisms(the second term of Eq.(2)). Similar considerations must be taken into account in comparing the PDG values with the predictions of other hadron structure calculations and in investigating the higher 
Table 2.1. Helicity amplitudes of the $\Delta \rightarrow \gamma N$ transition at $W=1236 \mathrm{MeV}$ are compared with the values from Particle Data Group (PDG) [10] and the predictions of constituent quark models of Refs. $[7,8]$. The amplitudes are in unit of $10^{-3}(\mathrm{GeV})^{-1 / 2}$.

\begin{tabular}{lccccc} 
A & PDG & Dressed & Bare & Ref. [8] & Ref. [7] \\
\hline$A_{3 / 2}$ & $-257 \pm 8$ & -228 & -153 & -157 & -186 \\
$A_{1 / 2}$ & $-141 \pm 5$ & -118 & -84 & -91 & -108
\end{tabular}

mass $N^{*}$ resonance parameters.

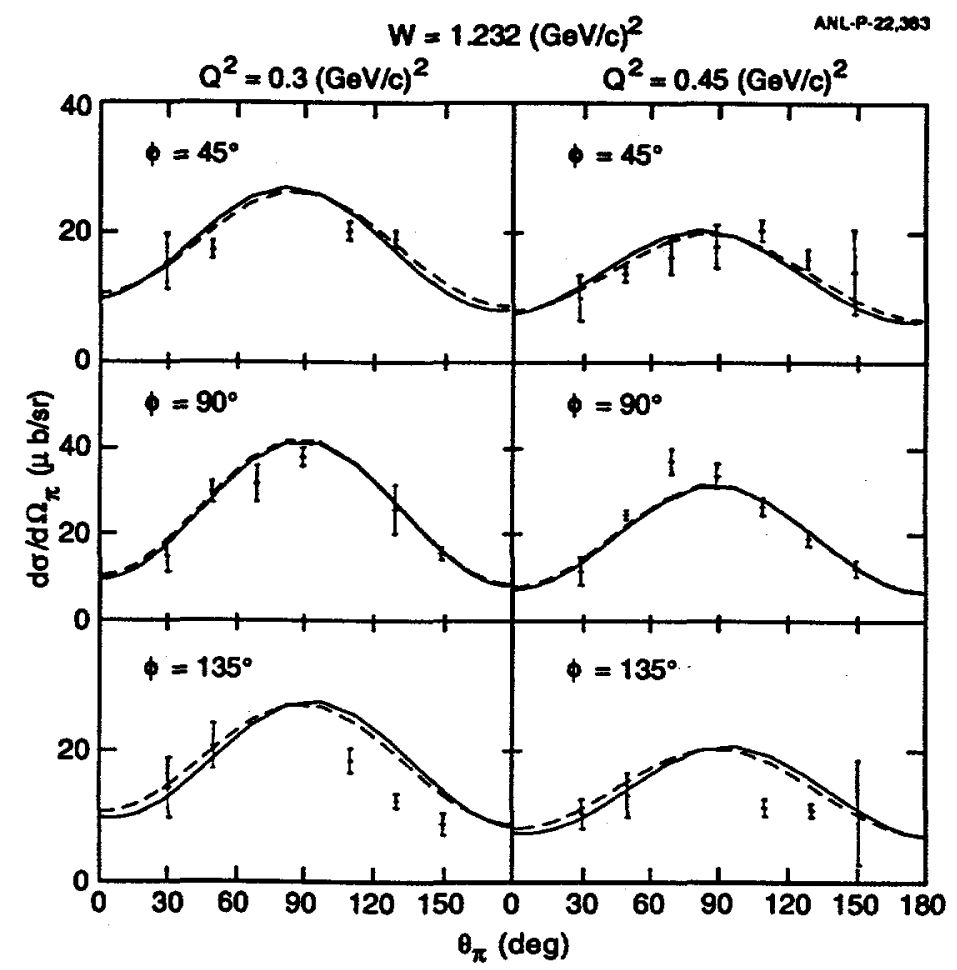

Fig. 2 The predicted differential cross sections of $p\left(e, e^{t} \pi^{0}\right)$ reactions are compared with the data.

The dynamical model of Ref.[5] has been extended to make predictions for $N\left(e, e^{\prime} \pi\right)$ reactions. As a first step to help the experimental efforts in the past few years, we had made predictions based on the simplest assumption that the electromagnetic form factors for the $\gamma N \rightarrow \Delta$ is proportional to the the well measured proton form factor. The charge component $G_{C}\left(q^{2}\right)$ of the $\gamma N \rightarrow \Delta$ form factor is calculated from $G_{M}\left(q^{2}\right)$ and $G_{E}\left(q^{2}\right)$ using the long wavelength approximation. In Fig.2, we see that our predictions are in good agreement with the available data. The comparison of our predictions of $A_{t l}=[d \sigma(\phi=$ $\left.0)-d \sigma\left(\phi=180^{\circ}\right)\right] /\left[d \sigma(\phi=0)+d \sigma\left(\phi=180^{\circ}\right)\right]$ with the most recent data from 
MIT-Bates are shown in Fig. 3. Here we see some significant deviations with the data at $W=1236 \mathrm{MeV}$. This could be mainly due to our use of the very naive form factors for the $\gamma N \rightarrow \Delta$ transition.

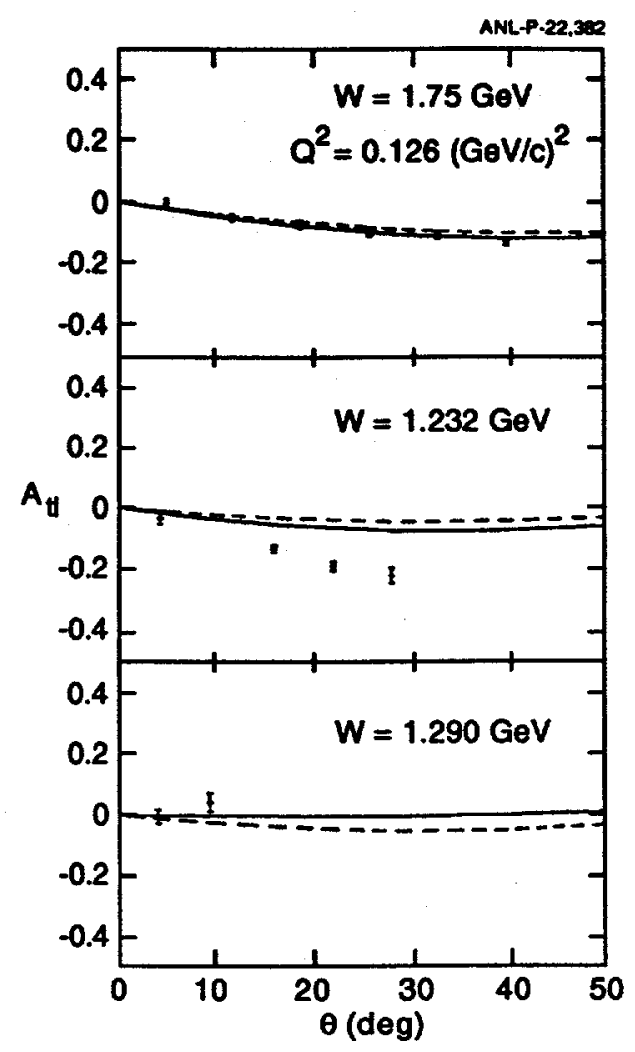

Fig.3 The predicted $A_{t l}$ are compared with the recent data from MIT-Bates.

\section{Chiral Constituent Quark Model Calculations}

Encouraged by the results of Ref.[5], as briefly described above, we[6] have started to apply our dynamical approach to test various constituent quark models in conjuction with the new experiments being conducted at MIT-Bates and Jefferson Laboratory. We start with the now well accepted assumption that the chiral constituent quark model can emerge from QCD due to the breaking of chiral symmetry. This is supported by a recent Lattice QCD calculation[11]. It is therefore reasonable to assume that a Hamiltonian for relating the $\pi N$ and $\gamma N$ reactions to the quark-substructure of $N$ and $\Delta$ is of the following form

$$
H=H_{B}+\sum_{B, B^{\prime}}\left[\left(h_{\pi B, B^{\prime}}+h_{\gamma B, B^{\prime}}\right)+(\text { h.c. })\right]
$$


where $B$ and $B^{\prime}$ are the eigenstates of an one-baryon Hamiltonian $H_{B}$. In the simplest nonrelativistic model, it is defined by

$$
H_{B}=\sum_{i}\left(m_{q}+\frac{p_{i}^{2}}{2 m_{q}}\right)+V+V_{\text {conf }}
$$

where $V_{\text {conf }}=\sum_{i>j} \alpha_{c} r_{i j}$ is the usual linear confinement potential. The quarkquark residual interaction $V$ is due to the exchange of Goldstone bosons and gluons. The vertex interactions in Eq.(6) are calculated from the baryon wavefunctions and quark operators

$$
\begin{aligned}
& f_{B, \pi B^{\prime}}=\left\langle B\left|\sum_{i} f_{\pi q, q}(i)\right| B^{\prime}\right), \\
& f_{B, \gamma B^{\prime}}=\left\langle B\left|\sum_{i} f_{\gamma q, q}(i)\right| B^{\prime}\right\rangle .
\end{aligned}
$$

In the simplest nonrelativistic model, the above quark-meson and quark-photon operators are well known and are used in our calculations. Within the dynamical approach of Ref.[5], the vertices defined by Eqs. (8)-(9) for $B=\Delta$ and $B^{\prime}=N$ are identified with the bare vertices $\Gamma_{\Delta, \gamma N}$ and $\Gamma_{\Delta, \pi N}$ in Eq.(1). Accordingly the $\Delta$ mass generated from the model Eq. (7) must be identified with the mass $1300 \mathrm{MeV}$ of the bare $\Delta$. To make contact with the previous works, we assume that the interactions between constituent quarks can be represented by the following form

$$
V=f_{G} V_{\text {oge }}+f_{M} V_{o m e},
$$

where $V_{\text {oge }}$ is the usual one-gluon-exchange(oge) potential and $V_{\text {ome }}$ is the onemeson-exchange(ome) potential due to the exchange of pion and eta mesons. Both the oge and ome potentials are regularized by a form factor of the form $F_{i}^{2}\left(q^{2}\right)=\left(\Lambda_{i}^{2} /\left(q^{2}+\Lambda_{i}^{2}\right)\right)$.

With the above formulation, we can investigate whether the $\pi N$ and $\gamma N$ reactions can distinguish three possible constituent quark models. For $f_{G}=1$ and $f_{M}=0$, the above equations define the traditional constituent quark model(oge). For $f_{G}=0$ and $f_{M}=1$ we have an one-meson-exchange model $(o m e)$ that is similar, but not identical, to the form proposed by Glozman and Riska[12]. The model with $f_{G}=f_{M}=1$ contains both the bosonexchange and gluon-exchange interactions between constituent quarks. This model (ogme) was briefly investigated in Ref.[14].

To be consistent with the dynamical model of Ref.[5], the parameters of each considered model must be chosen to reproduce (1) $m_{\Delta}-m_{N}=(1300$ - 938.5 ) MeV, (2) $G_{M}(0) \sim 2.0, G_{E}(0) \sim \pm 0.025$, (3) the magnetic moment $\mu_{p}$ of the proton, (4) the proton charge radius $\langle r\rangle_{p} \sim 0.8 \mathrm{fm}$, (5) the form factors $f_{\pi N, \Delta}(k)$ and $f_{\pi N, N}(k)$ with a cutoff of about $650 \mathrm{MeV} / \mathrm{c}$ in dipole form. By using the variational method developed in Ref.[15], we solve the threebody bound state problem by diagonalizing the model Hamiltonian, defined by 
Eq.(7), in the space spanned by the harmonic oscillator wavefunctions. The convergent solutions are obtained when up to about $\mathrm{N}=9$ orbitals are included. As a check of our calculation, we are able to reproduce the results of Glozman, Rapp and Plessas[13] if their short-range form of Goldstone boson exchange potential is used in our calculation.

We first adjust the parameters to fit the data (1)-(3) listed above. It turns out that all of the possible consitutent quark models fitted to these data yield a proton radius of only about $0.4 \mathrm{fm}$. Consequently, the predicted $\pi N N$ and $\pi N \Delta$ form factors are too hard in comparison with that of Ref.[5]. It is not clear how to remove this difficulty. One obvious possibility is to carry out a relativistic calculation and to consider interaction currents. Nevertheless, our results so far at least demonstrate that the $\pi N$ and $\gamma N$ reactions can be useful in distinguishing the three considered constituent quark models, since their predicted form factors have significant differences. This is illustrated in Fig.4 for the predicetd $N^{*}\left(S_{11}\right) \rightarrow \pi N, \eta N, \pi \Delta$ form factors.

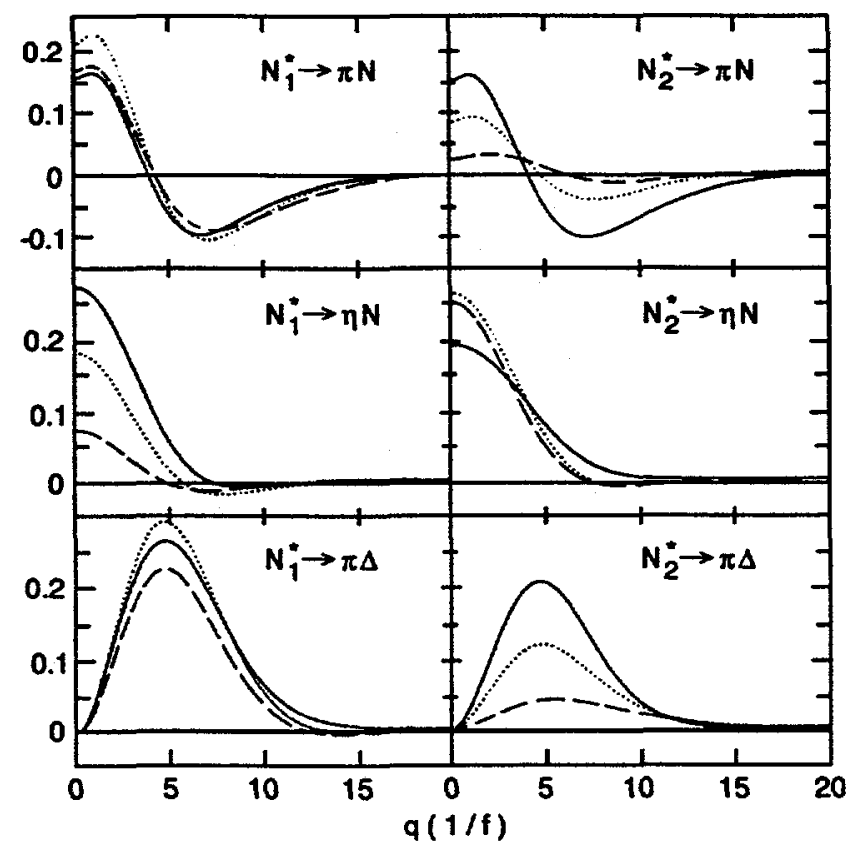

Fig.4 The $N^{*} \rightarrow \pi N, \eta N, \pi \Delta$ form factors. The solid, dotted and dashed curves are respectively from the oge, ome, and ogme models.

Clearly, much has to be done before we can use the $N\left(e, e^{\prime} \pi\right)$ data, like the data of $A_{t l}$ shown in Fig.3, to distinguish different constituent quark models. This is the focus of our current effort in order to confront the forthcoming data with high precision. This theory-experiment joint effort is needed to give 
a crucial test of the chiral constituent quark model and its dynamical content.

\section{4 summary}

We have reviewed the development of a dynamical approach to study the photoproduction and electroproduction of pions on the nucleon. Our current effort is to test the chiral constituent quark model which can be related to $Q C D$. Some preliminary results for the $N^{*}\left(S_{11}\right)$ have been presented to indicate that the predicted pion and photon form factors can be tested in the studies of $\pi N$ and $\gamma N$ reactions. Our complete results will be published elsewhere[6].

\section{References}

1. G.F. Chew, M.L. Goldberger, F.E. Low and Y. Nambu, Phys. Rev. 106(1957) 1345.

2. O. Hanstein, D. Drechsel and L. Tiator, Nucl. Phys. A 632(1998) 561.

3. R.M. Davidson, N.C. Mukhopadhyay and R.S. Wittman, Phys. Rev. D 43 (1991) 71, and references therein.

4. V. Bernard, N. Keiser and U. Meissner, Phys. Lett. 382 , 19 (1996), and references therein

5. T. Sato and T.-S. H. Lee, Phys. Rev. C 54 (1996) 2660, and references therein.

6. T. Yoshimoto, T.Sato, M. Arima and T.-S. H. Lee, in preparartion.

7. S. Capstick, Phys. Rev. D46, 2864 (1992).

8. R. Bijker, F. Iachello, and A. Leviatan, Ann. of Phys. 236, 69 (1994).

9. LEGS Collaboration, A.M. Sandorfi et al., Few Body Systems, Suppl. 7 (1994) 317; LEGS Data Release L7a8.0 (April '94); A.M. Sandorf, private communication.

10. Review of Particle Properties, L. Montanet et al., Phys. Rev. D 50 (1994) 1710

11. K.F. Liu, talk at Trento workshop on "Nonperturbative QCD and $N^{*}$ Physics", ECT, May 1998.

12. L. Ya. Glozman and D.O. Riska, Phys. Rep. 268. 263 (1996).

13. L. Ya. Glozman, Z. Rapp, and W. Plessas, Phys. Lett. B381, 311 (1996)

14. P.N. Shen, Y.-B. Dong, Z.-Y. Zhang, Y.-W. Yu and T.-S. H. Lee, Phys. Rev. C55, 2024 (1997).

15. T. Ogaito, M. Arima and and T. Sato, to be published. 